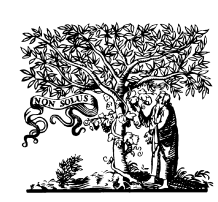

ELSEVIER

NDT\&E International 32 (1999) 293-303

\title{
Analysis of eddy currents in a bar containing an embedded defect
}

\author{
J.W. Liaw ${ }^{\mathrm{a}}$, S.L. Chu ${ }^{\mathrm{a}}$, C.S. Yeh ${ }^{\mathrm{b}}$, M.K. Kuo ${ }^{\mathrm{b}}$ \\ ${ }^{a}$ Materials Research Laboratories, Industrial Technology Research Institute, Bldg. 77, 195-5 Chung Hsing Rd., Section 4 Chutung, Hsinchu, Taiwan, ROC \\ ${ }^{\mathrm{b}}$ Institute of Applied Mechanics, National Taiwan University, Taipei, Taiwan, ROC
}

Received 25 October 1997; received in revised form 7 May 1998; accepted 14 July 1998

\begin{abstract}
The eddy current distribution is studied in a metal bar containing an embedded defect inserted within an encircling coil. Furthermore, the impedance change of the coil regarding the characteristics of the defect is determined. The defects investigated are nonmetallic inclusions and embedded cracks which may occur during the manufacturing processes of bars. To simulate these problems, a two-dimensional transverse-electric model is proposed, and then a set of coupled surface integral equations are formulated systematically. Since the magnetic field is unknown along the boundary of the defect, an additional boundary condition derived from Maxwell's equations is used. Using the boundary element method (BEM), these integral equations are solved in terms of nodal unknowns-current density. After the current distribution is obtained, the impedance changes of the coil caused by the defects are calculated vs. the configuration of defect for various frequencies. If the inclusion is circular and located at the center of the bar, good agreements are found by comparing the BEM solutions with the analytical ones. An auxiliary surface integral equation is also derived to further determine the currents on both sides of the crack. C 1999 Elsevier Science Ltd. All rights reserved.
\end{abstract}

Keywords: Eddy currents; Cracks; Inclusion; Boundary element method

\section{Introduction}

Eddy current testing (ECT) has been widely used for product evaluation in steel industries. One important application is to detect nondestructively the surface and subsurface defects of steel bar and wire by ECT [1]. The defects could be cracks or nonmetallic inclusions, e.g. oxide. As we know, bars and wires are used as raw material for the manufacture of various mechanical parts, such as nuts, screws, bolts, shafts, etc. Therefore, these defects are not tolerated, when their lengths exceed a specific value. If the bars are ferromagnetic materials, such as carbon-steel, they always need to be magnetized to saturation to reduce the effect of permeability variation during the off-line inspection. In the on-line inspection of hot rolling, since the temperature of the bar is above the Curie temperature, the texture of carbon-steel is without ferromagnetic property. Therefore we do not consider the hysteresis behavior in this paper for actual application. Another application of the eddy current is using a coil to induce eddy current to generate Joule heat $[2,3]$ in a billet. Due to the presence of defects, the current distribution changes, and the temperature distribution on the billet's surface deviates from normal. Thus, using infra-red sensors to scan the billet, one can detect and locate the surface and sub-surface defects.

The principle of ECT is that a flaw in a conductor, as an obstacle for eddy currents, perturbs the distribution of current in the conductor and the electromagnetic field in space so as to change the impedance of the coil. This means we can evaluate defects in metals according to impedance changes whose values are related to the depth, length and orientation of the defect [4-10]. Hower et al. [11] used the integral equation of magnetic and electric field to solve the three-dimensional embedded defects problem, but the essential limitation is the low frequency assumption. Beissner [12] studied the effect of flaws on eddy currents using the boundary element method (BEM). Kahn [13] used BEM to investigate the current distribution in a half-plane with an inclined surface crack. Bowler [14] solved a boundary integral equation in terms of current dipoles to analyze a halfspace with a sub-surface crack. In ECT, the probes, encircling coils, are usually used to detect the surface and subsurface defects of metal bars at a fixed frequency. Dodd and Deeds [15] gave a close-form solution for the interaction between an encircling coil and a bar, an axial-symmetric problem. Forster [1] used a mercury model, a tube full of mercury and encircled by a coil, to measure experimentally the impedance change of the coil by a crack. The corresponding problem generally is a three-dimensional one, due to the configuration of coil, crack, and bar. However if we assume the axial lengths of solenoid coil, bar, and longitudinal defect all are infinitely long, and their axial 

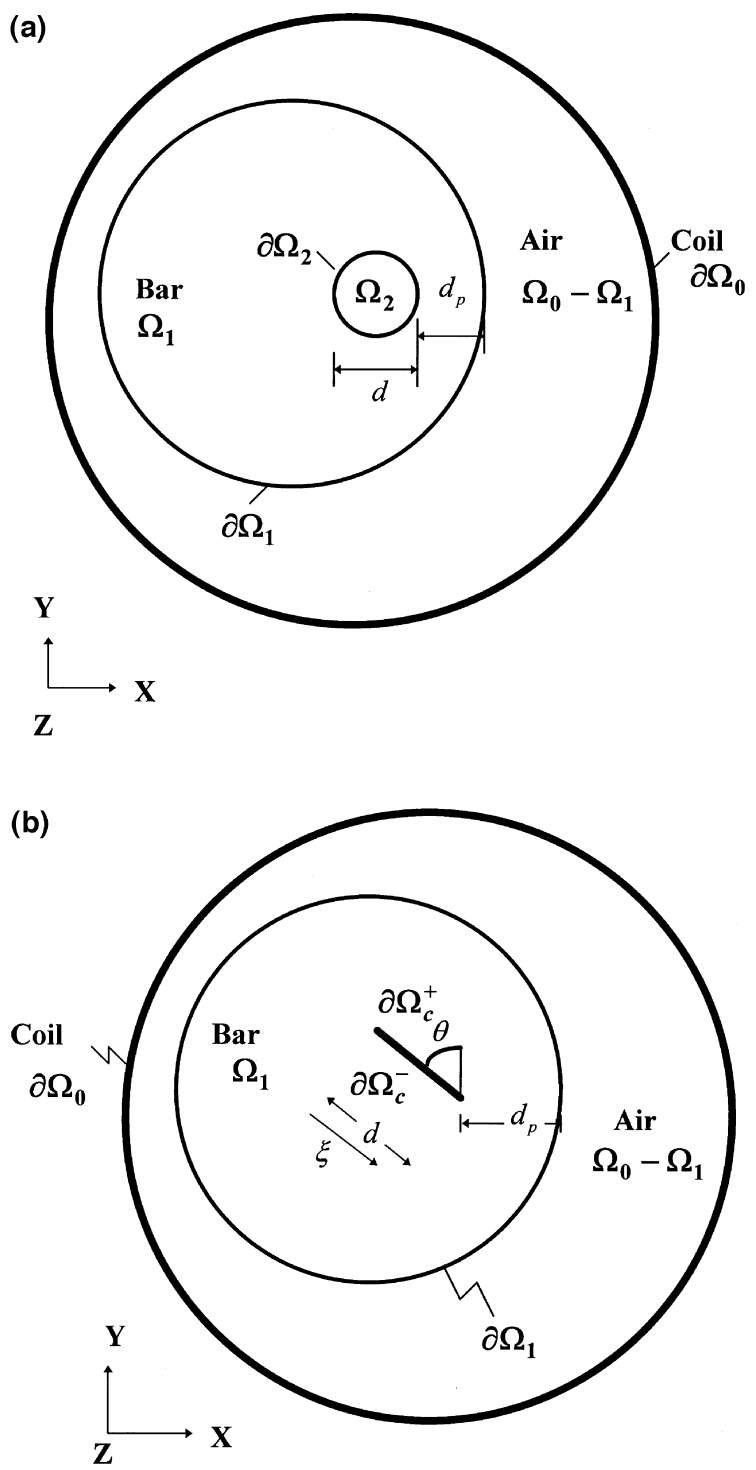

Fig. 1. (a) The cross-sectional view of a bar, with a nonmetallic inclusion, placed within an encircling coil; (b) Cross-sectional view of a bar, with an embedded crack, placed within an encircling coil (coordinate $\xi \in[0, d]$ ).

orientations are parallel to the $z$ direction, the problem is reduced to a two-dimensional, transverse-electric one. For this problem, Spal and Kahn [16] used an analytical method to study a cylinder containing a vertical surface crack. Later Kahn and Spal [17] formulated an integral equation which is solved numerically by BEM. Chari and Kincaid [18] also studied this problem using the finite element method (FEM). After that, Brudar [19] employed the finite difference method (FDM) to analyze this problem.

In this paper, we study the behavior of eddy currents in a bar with an embedded defect, and utilize the results to calculate the impedance change of a coil, an essential parameter of ECT. The defects investigated in this multiply-connected problem are nonmetallic inclusions and embedded cracks which may occur during the manufacturing processes. This induction problem is formulated by a set of surface integral equations solved by BEM. The significant difficulty encountered is that the magnetic field inside the embedded flaw is unknown, and is proved to be uniform. Hence an additional boundary condition along the defect is derived. The embedded crack investigated is assumed to be closed, i.e. there is no crack opening displacement. This assumption is reasonable, because all the voids will collapse to seams during the rolling of bars. After the eddy current distributions on the surface of the conductor as well as the flaw are obtained, the impedance change of the coil caused by the flaw is determined versus the depth, length, and orientation of flaw for various frequencies. If the shape of the inclusion is circular and located at the center of the bar, we provide analytical solutions to compare with BEM solutions, and good agreement is found. In addition, an auxiliary surface integral equation is derived to determine the currents on both sides of the crack. To reduce the number of parameters for each problem, all parameters and variables of numerical results are presented in non-dimensional form.

\section{Integral equations for TE mode}

In this section, the integral representations of magnetic field and current density in conductors as well as the integral equations of the surface components are derived systematically. Conductors with an embedded flaw, inclusion and crack, are studied. Consider a metal bar with an arbitrary cross-section placed within a solenoid-type encircling coil. The axial lengths of bar, flaw and coil are infinitely long, and their axial orientations are parallel to the $z$ direction. The cross-sectional views with different flaws are shown in Fig. 1(a,b). These are two-dimensional transverse-electric (TE) mode problems, because the magnetic field is $\mathbf{H}=H_{z} \mathbf{e}_{z}$, where $\mathbf{e}_{z}$ is the unit vector of $z$ direction, and all functions are independent of $z$. In this paper, we assume that a sinusoidal current with constant amplitude $I_{0}$ is imposed on the coil to induce an eddy current in the metal bar, and that all magnetic flux is confined within the coil. Hence, the interrogating magnetic strength field inside the coil but outside the conductor is uniform, $H_{z}=n I_{0}$, where $n$ is the winding turns per unit length of coil. For simplicity, the common time harmonic factor $\exp (j \omega t)$ is omitted in the following equations. It is assumed that the material properties of the conductor are linear, isotropic, and homogeneous with constant permeability $\mu$ and constant electrical conductivity $\sigma$. The current density $\mathbf{J}$ in conductors obeys Ohm's law, $\mathbf{J}=\sigma \mathbf{E}$, where $\mathbf{E}$ is the electric field, so that $\nabla \times \mathbf{H}=\left(\sigma+j \omega \varepsilon_{0}\right) \mathbf{E}$. Here, $\varepsilon_{0}$ is the dielectric constant of metal. Furthermore, we assume there is no contribution of charge density in conductors. As a result, from Maxwell's equations, the integral representations of $\mathbf{E}$ and magnetic 
field $\mathbf{H}$ in the conductor are given by

$$
\begin{aligned}
\mathbf{H}= & -\left(\sigma+j \omega \varepsilon_{0}\right) \int_{\partial \Omega}(\mathbf{n} \times \mathbf{E}) G \mathrm{~d} s-\int_{\partial \Omega}(\mathbf{n} \times \mathbf{H}) \nabla G \mathrm{~d} s \\
& -\int_{\partial \Omega}(\mathbf{n} \times \mathbf{H}) \times \nabla G \mathrm{~d} s, \\
\mathbf{E}= & j \omega \mu \int_{\partial \Omega}(\mathbf{n} \times \mathbf{H}) G \mathrm{~d} s-\int_{\partial \Omega}(\mathbf{n} \times \mathbf{E}) \nabla G \mathrm{~d} s \\
& -\int_{\partial \Omega}(\mathbf{n} \times \mathbf{E}) \times \nabla G \mathrm{~d} s,
\end{aligned}
$$

which are Stratton-Chu formula [20], where no volume integral exists. $\Omega$ represents the region of the conductor and $\partial \Omega$ denotes its boundary. In the above equations, $\mathbf{n}$ is the unit normal vector out of the conducting medium. Green's function $G$, the singular solution of Helmholtz equation, satisfies

$\nabla^{2} G+\left(\omega^{2} \varepsilon_{0} \mu-j \omega \sigma \mu\right) G=-\delta\left(\mathbf{x}-\mathbf{x}^{\prime}\right)$,

where $\delta\left(\mathbf{x}-\mathbf{x}^{\prime}\right)$ denotes the Dirac delta function. For the two-dimensional problems with an interior region, Green's function is given by

$G\left(\mathbf{x}, \mathbf{x}^{\prime}\right)=-\frac{1}{4} Y_{0}\left(k\left|\mathbf{x}-\mathbf{x}^{\prime}\right|\right)$,

where $Y_{0}$ is the Bessel function of the second kind of order zero. Since, for a quasi-static problem, the displacement current whose order is much less than the order of eddy current can be neglected, the complex wave number $k$ is given by

$k=\frac{1-j}{\delta}$,

in terms of the eddy current penetration depth $\delta$

$\delta=1 / \sqrt{\pi \sigma \mu f}$

where $f=\omega / 2 \pi$. Moreover, for this 2-D TE-mode, Eq. (1) is reduced to the integral representation for $H_{z}$ as

$$
\begin{aligned}
H_{z}(\mathbf{x})= & -\int_{\partial \Omega} H_{z} \mathbf{n}\left(\mathbf{x}^{\prime}\right) \cdot \nabla^{\prime} G\left(\mathbf{x}, \mathbf{x}^{\prime}\right) \mathrm{d} l^{\prime}-\int_{\partial \Omega} J_{s} G\left(\mathbf{x}, \mathbf{x}^{\prime}\right) \mathrm{d} l^{\prime}, \\
& \mathbf{x} \in \Omega,
\end{aligned}
$$

where the displacement current is neglected and the relation, $-\left(\mathbf{e}_{z} \times \mathbf{n}\right) \times \nabla^{\prime}=\mathbf{n} \cdot \nabla^{\prime}$, is used. The 2 -D gradient is defined as $\nabla^{\prime}=\mathbf{e}_{x} \partial / \partial x^{\prime}+\mathbf{e}_{y} \partial / \partial y^{\prime}$. If the boundary is insulated, since no normal component of the current is on boundary, the current density of the surface is $J_{s}=\sigma E_{t}$. Here, $E_{t}$ is the tangential component of electric field on the surface, namely $E_{t}=\mathbf{E} \cdot \mathbf{t}$. The tangential vector, $\mathbf{t}$, is the unit vector along the surface of cross-section of bar, $\mathbf{t}=\mathbf{e}_{z} \times \mathbf{n}$. Since, for TEmode problem $H_{z}$ satisfies scalar Helmholtz equation, Eq. (6) can also be directly derived by Green's theorem [17], with $\partial H_{z} / \partial n=-J_{s}, \mathbf{x} \in \partial \Omega$.
Since the surface $\partial \Omega$ allows no normal component of current to pass through, the integral representation of electric field for TE-mode is derived from Eq. (2) as

$\mathbf{E}(\mathbf{x})=-\int_{\partial \Omega} j \omega \mu H_{z} G \mathrm{~d} \mathbf{l}^{\prime}-\int_{\partial \Omega} E_{t} \mathbf{e}_{z} \times \nabla^{\prime} G \mathrm{~d} l^{\prime}, \quad \mathbf{x} \in \Omega$,

where $\mathbf{n} \times \mathbf{E}=0, \mathbf{n} \times \mathbf{H}=H_{z} \mathbf{t},(\mathbf{n} \times \mathbf{E}) \times \nabla^{\prime} G=E_{t} \mathbf{e}_{z}$ $\times \nabla^{\prime} G$, are used. Multiplying Eq. (7) by $\sigma$, the integral representation for current density is obtained

$\mathbf{J}(\mathbf{x})=-\int_{\partial \Omega} j \omega \mu \sigma H_{z} G \mathrm{~d} \mathbf{l}^{\prime}-\int_{\partial \Omega} J_{s} \mathbf{e}_{z} \times \nabla^{\prime} G \mathrm{~d} l^{\prime}, \quad \mathbf{x} \in \Omega$.

To study the effect of embedded flaws, nonmetallic inclusions and longitudinal cracks, the integral equations for a conductor containing a flaw are further derived. In particular, for the conductors with a closed crack, the integral equations will reduce to special ones, due to the degenerated geometry. Since the cross-sections of bars with an embedded flaw are multiply-connected domains, one extra condition is also required for the flaw, an inclusion or a crack, as illustrated in the following.

\subsection{Bar with a nonmetallic inclusion}

The boundary of a bar with a nonmetallic inclusion or void is $\partial \Omega=\partial \Omega_{1} \cup \partial \Omega_{2}$, where $\partial \Omega_{1}$ denotes the circumference of the bar, and $\partial \Omega_{2}$ the boundary of the inclusion. The cross-sectional view of the bar is depicted in Fig. 1(a). If the boundary is smooth, as $\mathbf{x}$ approaches the boundary, the integral equation of magnetic field, from Eq. (6), is

$$
\begin{gathered}
\frac{1}{2} H_{z}(\mathbf{x})=-\int_{\partial \Omega_{1} \cup \partial \Omega_{2}} H_{z} \mathbf{n}\left(\mathbf{x}^{\prime}\right) \cdot \nabla^{\prime} G \mathrm{~d} l^{\prime}-\int_{\partial \Omega_{1} \cup \partial \Omega_{2}} J_{s} G \mathrm{~d} l^{\prime}, \\
\quad \mathbf{x} \in \partial \Omega_{1} \cup \partial \Omega_{2} .
\end{gathered}
$$

The first integral of the right-hand side of Eq. (9) takes the Cauchy principal value. The tangential direction of the outer surface $\partial \Omega_{1}$ follows the counterclockwise direction, and that of the inner surface $\partial \Omega_{2}$ follows the clockwise direction. As $\mathbf{x}$ approaches the surface, taking the inner product of Eq. (8) with $\mathbf{t}$, we obtain the surface integral equation for $J_{s}$, after some manipulation

$$
\begin{aligned}
\frac{1}{2} J_{s}(\mathbf{x})= & -j \omega \int_{\partial \Omega_{1} \cup \partial \Omega_{2}} \sigma \mu H_{z} G \mathbf{n}(\mathbf{x}) \cdot \mathbf{n}\left(\mathbf{x}^{\prime}\right) \mathrm{d} l^{\prime} \\
& -\int_{\partial \Omega_{1} \cup \partial \Omega_{2}} J_{s} \mathbf{n}(\mathbf{x}) \cdot \nabla^{\prime} G \mathrm{~d} l^{\prime}, \\
& \mathbf{x} \in \partial \Omega_{1} \cup \partial \Omega_{2},
\end{aligned}
$$

where the relation $\mathbf{t} \cdot\left(\mathbf{e}_{z} \times \nabla^{\prime}\right)=\mathbf{n} \cdot \nabla^{\prime}$ is used. Eq. (9) is the Fredholm integral equations of the first kind for unknown $J_{s}$, and is that of the second kind for $H_{z}$. While Eq. (10) is that of the first kind for $H_{z}$, and of the second kind for $J_{s}$. For 
numerical computations, since $H_{z}$ is given on $\partial \Omega_{1}$, either Eq. (9) or Eq. (10) can be chosen as the governing equation of $J_{s}$. Therefore Eq. (10) is equivalent to Eq. (9) for calculating $J_{s}$ as $\mathbf{x} \in \partial \Omega_{1} \cup \partial \Omega_{2}$. Subsequently, we should discuss the magnetic field in the inclusion. Because the nonmetallic inclusion or void is not a conductor, no current can pass through these defects. Since the displacement current is neglected, the magnetic field in the inclusion satisfies $\nabla \times \mathbf{H}=0$, which leads to $\partial H_{z} / \partial x=0, \partial H_{z} /$ $\partial y=0$. Therefore the magnetic field in the inclusion is easily proven a constant, denoted by $H_{z}^{(2)}$. However, Eqs. (9) and (10), for a conductor, cannot provide sufficient conditions to determine this constant magnetic field within the inclusion. To overcome this problem, we derive an additional condition using the integral form of Maxwell's equation, $\nabla \times \mathbf{E}=-j \omega \mathbf{B}$, for the inclusion

$-\int_{\partial \Omega_{2}} J_{s} \mathrm{~d} l=-j \omega \sigma \mu_{o} \mu_{r 2} A_{2} H_{z}^{(2)}$,

where $A_{2}, \mu_{r 2}, H_{z}^{(2)}$ correspond to the cross-sectional area of the inclusion, its relative permeability, and the constant magnetic field within it, respectively. Physically, Eq. (11) states that the line integral of current around the flaw is proportional to the net magnetic flux through the area of the flaw perpendicularly. This additional condition can be regarded as the governing equation of the nonmetallic inclusion. With the aid of Eq. (11), either Eq. (9) or Eq. (10) is chosen for solving $J_{s}, H_{z}^{(2)}$. After the distributions of $J_{s}, H_{z}^{(2)}$ are determined, the fields $H_{z}, \mathbf{E}$, and $\mathbf{J}$ in the conductor can be calculated using the integral representation of Eqs. (6-8), respectively.

\subsection{Bar with an embedded crack}

For a bar with a longitudinal embedded crack, its boundary is $\partial \Omega=\partial \Omega_{1} \cup \partial \Omega_{c}$. The embedded crack is assumed to be a mathematical line submerged in a bar and its boundary is $\partial \Omega_{c}=\partial \Omega_{c}^{+} \cup \partial \Omega_{c}^{-}$, as shown in Fig. 1(b), i.e. the crack is closed without crack opening displacement. We can regard this crack as a squeezed void. That is to say the upper surface $\partial \Omega_{c}^{+}$coincides with the lower surface $\partial \Omega_{c}^{-}$. Moreover, the crack is assumed to be an insulator which allows no current to pass through it, i.e. $\mathbf{J} \cdot \mathbf{n}^{ \pm}=0$. As $\mathbf{x}$ approaches the surface, the integral equation of magnetic field is derived from Eq. (6) as

$$
\begin{aligned}
\frac{1}{2} H_{z}(\mathbf{x})= & -\int_{\partial \Omega_{1}} H_{z} \mathbf{n}\left(\mathbf{x}^{\prime}\right) \cdot \nabla^{\prime} G \mathrm{~d} l^{\prime}-\int_{\partial \Omega_{1}} J_{s} G \mathrm{~d} l^{\prime} \\
& -\int_{\partial \Omega_{c}^{+}}\left[J_{s}\right] G \mathrm{~d} l^{\prime}, \\
& \mathbf{x} \in \partial \Omega_{1},
\end{aligned}
$$

$$
\begin{aligned}
H_{z}(\mathbf{x})= & -\int_{\partial \Omega_{1}} H_{z} \mathbf{n}\left(\mathbf{x}^{\prime}\right) \cdot \nabla^{\prime} G \mathrm{~d} l^{\prime}-\int_{\partial \Omega_{1}} J_{s} G \mathrm{~d} l^{\prime} \\
& -\int_{\partial \Omega_{c}^{+}}\left[J_{s}\right] G \mathrm{~d} l^{\prime}, \\
& \mathbf{x} \in \partial \Omega_{c},
\end{aligned}
$$

where the discontinuity of current is $\left[J_{s}\left(\mathbf{x}^{\prime}\right)\right]$ $=J_{s}^{+}\left(\mathbf{x}^{\prime}\right)+J_{s}^{-}\left(\mathbf{x}^{\prime}\right)=\sigma\left(\mathbf{E}^{+} \cdot \mathbf{t}^{+}+\mathbf{E}^{-} \cdot \mathbf{t}^{-}\right), \mathbf{x}^{\prime} \in \partial \Omega_{c}$. The superscripts + , and - denote the upper and the lower surface of the crack, respectively, and $\mathbf{t}^{-}=-\mathbf{t}^{+}, \mathbf{n}^{-}=-\mathbf{n}^{+}$. We can also derive the integral equation of current from Eq. (8) as follows

$$
\begin{aligned}
\frac{1}{2} J_{s}(\mathbf{x})= & -j \omega \int_{\partial \Omega_{1}} \sigma \mu H_{z} G \mathbf{n}(\mathbf{x}) \cdot \mathbf{n}\left(\mathbf{x}^{\prime}\right) \mathrm{d} l^{\prime} \\
& -\int_{\partial \Omega_{1}} J_{s} \mathbf{n}(\mathbf{x}) \cdot \nabla^{\prime} G \mathrm{~d} l^{\prime}-\int_{\partial \Omega_{c}^{+}}\left[J_{s}\right] \mathbf{n}(\mathbf{x}) \cdot \nabla^{\prime} G \mathrm{~d} l^{\prime} \\
& \mathbf{x} \in \partial \Omega_{1},
\end{aligned}
$$

$$
\begin{aligned}
\frac{1}{2}\left\langle J_{s}(\mathbf{x})\right\rangle= & -j \omega \int_{\partial \Omega_{1}} \sigma \mu H_{z} G \mathbf{n}^{+}(\mathbf{x}) \cdot \mathbf{n}\left(\mathbf{x}^{\prime}\right) \mathrm{d} l^{\prime} \\
& -\int_{\partial \Omega_{1}} J_{s} \mathbf{n}^{+}(\mathbf{x}) \cdot \nabla^{\prime} G \mathrm{~d} l^{\prime}-\int_{\partial \Omega_{c}^{+}}\left[J_{s}\right] \mathbf{n}^{+}(\mathbf{x}) \cdot \nabla^{\prime} G \mathrm{~d} l^{\prime}, \\
& \mathbf{x} \in \partial \Omega_{c},
\end{aligned}
$$

where $\left\langle J_{s}\right\rangle=J_{s}^{+}-J_{s}^{-}$. For numerical computation, Eq. (13a) is equivalent to Eq. (12a) as $\mathbf{x} \in \partial \Omega_{1}$. Regardless of $\mathbf{x} \in$ $\partial \Omega_{c}^{-}$or $\mathbf{x} \in \partial \Omega_{c}^{+}$, Eqs. (12b) and (13b) are the only forms which we have. In general, $\partial \Omega_{c}$ is an arc-curve. If the crack is a straight line, the third term of the right-hand side of Eq. (13b) vanishes, due to $\mathbf{n}^{+} \perp \mathbf{x}-\mathbf{x}^{\prime}$, as $\mathbf{x}, \mathbf{x}^{\prime} \in \partial \Omega_{c}$. Moreover, if $\partial \Omega_{1}$ is a circle and the crack is a straight line with $\theta=90^{\circ}$, the current distribution in the bar is symmetric with respect to the crack, leading to $\left\langle J_{s}\right\rangle=0$.

Subsequently, an addition condition for determining the magnetic field in the crack is derived. First the crack is assumed opened, i.e. the area of crack is not equal to zero. Since this crack can be regarded as a squeezed void, the same as a nonmetallic inclusion, the magnetic field inside the opened crack is also proven a constant, denoted by $H_{z}^{(c)}$. Furthermore, we assume the magnetic field inside the crack remains bounded, as the area of crack approaches zero to be closed. In the following, we will show the numerical results confirm this assumption that $H_{z}^{(c)}$ is finite. As a consequence, the additional condition (Eq. 11) is degenerated to

$\int_{\partial \Omega_{c}^{+}}\left[J_{s}\right] \mathrm{d} l=0$,

due to no crack opening displacement. The above equation illustrates that the line integral of current flow around the 
embedded crack is equal to zero, i.e. there is no net magnetic flux through it. The additional condition (Eq. 14) can be regarded as the governing equation for the embedded crack. Although this condition (Eq. 14) does not include the magnetic field, $H_{z}^{(c)}$, we can use it with the governing equations of the conductor to solve the magnetic field and the current around the crack. Therefore, either Eq. (12a) or Eq. (13a) is chosen to combine with Eqs. (12b) and (14) to solve the surface current density $J_{s}$ on $\partial \Omega_{1}$ and $\left[J_{s}\right], H_{z}^{(c)}$ on $\partial \Omega_{c}$. Subsequently, if we want to know the current density on each side of crack in detail, Eq. (13b) supplies an auxiliary integral equation. Substituting $J_{s},\left[J_{s}\right], H_{z}^{(c)}$ into Eq. (13b), the value $\left\langle J_{s}\right\rangle$ on $\partial \Omega_{c}$ is calculated. Then the values of $J_{s}^{+}, J_{s}^{-}$can straightforwardly be gained from $\left[J_{s}\right],\left\langle J_{s}\right\rangle$.

\section{Impedance of the coil}

By the electromagnetic interaction between coil, conductor, and flaw, the distribution of eddy currents will affect the input impedance of the coil at a given frequency. Therefore, once the distribution of surface current is determined, one can calculate the coil's impedance, which is the measured parameter in ECT, for presenting the inductive behavior between the coil and the medium. Since an alternating current with constant amplitude is applied to the coil, the impedance value $Z$ per unit length of the coil with embedded flaw, by definition, is expressed as

$$
\begin{aligned}
& Z=\frac{V}{I}=\frac{-n}{I} \int_{\partial \Omega_{o}} \mathbf{E} \cdot \mathrm{d} \mathbf{l} \\
& =\frac{-n}{I}\left(-j \omega \int_{\Omega_{o}-\Omega_{1}} \mathbf{B} \cdot \mathrm{d} \mathbf{a}+\int_{\partial \Omega_{1}} \mathbf{E} \cdot \mathrm{d} \mathbf{l}\right) \\
& =\frac{-n}{I_{o}}\left(-j \omega \mu_{o} n I_{o}(1-\eta) A+\frac{1}{\sigma} \int_{\partial \Omega_{1}} J_{s} \mathrm{~d} l\right),
\end{aligned}
$$

where the fill factor $\eta$ is the ratio of the cross-sectional area of bar to that of coil, namely $A$. If $\eta$ equals unity, the first term of the above equation vanishes. The region $\Omega_{0}, \Omega_{1}$, $\Omega_{0}-\Omega_{1}$ are the cross-sections of coil, bar, and air, respectively. The line integral along $\partial \Omega_{1}$ is in the counterclockwise sense. We define the impedance change as the difference between impedance in the presence and the absence of a flaw. Consequently, the impedance change $\Delta Z=Z-Z_{o}$ caused by the embedded flaw is given by

$\Delta Z=\frac{-n}{\sigma I_{o}} \int_{\partial \Omega_{1}} \Delta J_{s} \mathrm{~d} l$,

where $\Delta J_{s}=J_{s}-J_{s o}$. Here, $Z_{o}, J_{s o}$ correspond to the impedance and current density on the surface of an unflawed bar. The above equation is available for any kind of embedded defect. However the distribution of eddy current depends on the position and shape of the flaw. Eq. (16) states that only the change of current, $\Delta J_{s}$, on the circumference of the bar contributes to the impedance change. There is a little difference with a surface crack problem, in which $\Delta J_{s}$ as well as the additional current, $\left[J_{s}\right]$, on the crack surface contribute to the impedance change [13]. According to Lorentz reciprocity theorem of two-dimensions, we have

$\int_{\partial \Omega}\left(\mathbf{E}_{0} \times \mathbf{H}-\mathbf{E} \times \mathbf{H}_{0}\right) \cdot \mathbf{n} \mathrm{d} l=0$,

where $\mathbf{E}_{0}, \mathbf{H}_{0}$ are the electric and magnetic field of the unflawed bar, and $\mathbf{E}, \mathbf{H}$ are that of the flawed bar. The boundary $\partial \Omega$ is $\partial \Omega=\partial \Omega_{1} \cup \partial \Omega_{2}$ for an inclusion, or $\partial \Omega=\partial \Omega_{1} \cup \partial \Omega_{c}$ for an embedded crack. Using Eq. (17), Eq. (16) can be expressed in another form for the inclusion problem

$\Delta Z=\frac{1}{\sigma I_{o}^{2}} \int_{\partial \Omega_{2}}\left(J_{s} H_{z o}-\left(\sigma \mathbf{E}_{o} \cdot \mathbf{t}\right) H_{z}^{(2)}\right) \mathrm{d} l$.

Eq. (18) had already been obtained by Auld et al. [21]. For the embedded closed crack problem, Eq. (18) becomes

$\Delta Z=\frac{1}{\sigma I_{o}^{2}} \int_{\partial \Omega_{c}^{+}}\left[J_{s}\right] H_{z o} \mathrm{~d} l$.

In fact, real measurement systems of ECT apply a source with constant amplitude sinusoidal voltage instead of current to the coil. However, the impedance of the coil, a function of frequency, is an intrinsic property regardless of the exciting method. Therefore the impedance change calculated by Eq. (16) can be utilized to simulate the real measurement.

\section{Dimensionless treatment}

The above equations are available for arbitrary shape of inclusion and any curve of crack. For simplicity, we only consider the circular inclusion and straight line crack in the following. Bars with circular cross-sections are considered. In order to present the problems clearly, all terms are reduced to the non-dimensional forms, denoted by a superscript '*', as follows

$f^{*}=f / f_{g}$,

$d^{*}=d / R$,

$d_{p}^{*}=d_{p} / R$,

$H_{z}^{*}=H_{z} / n I_{o}$,

$J_{s}^{*}=J_{s} R / n I_{o}$,

$Z^{*}=Z / \omega L_{o}$,

$\mathbf{x}^{*}=\mathbf{x} / R$

where the limit frequency is $f_{g}=\left(2 \pi \sigma \mu R^{2}\right)^{-1}$ [1]. Here, $R$ is the radius of the bar, $d$ corresponds to the diameter of circular inclusion or the length of embedded crack, as 


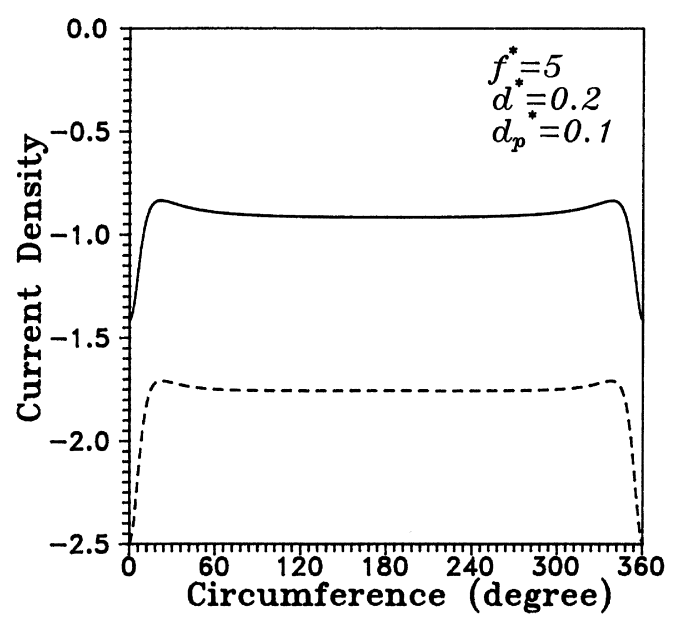

(a)

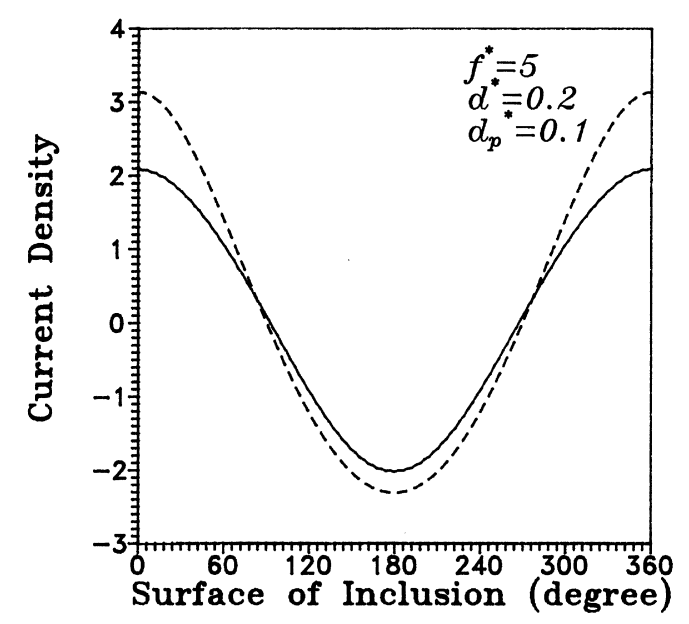

(b)

Fig. 2. (a) Distribution of $J_{s}^{*}$ on circumference $\partial \Omega_{1}$ for $f^{*}=5, d^{*}=0.2$, $d_{p}^{*}=0.1 . H_{z}^{(2) *}=(0.838,-0.260), \Delta Z^{*} / \eta \mu_{r}=\left(-2.403 \times 10^{-3}, 1.003 \times\right.$ $10^{-2}$ ). The solid line denotes $\operatorname{Re}\left(J_{s}^{*}\right)$, and the dashed line $\operatorname{Im}\left(J_{s}^{*}\right)$ (circular nonmetallic inclusion); (b) Distribution of $J_{s}^{*}$ on the surface of the inclusion. The solid line denotes $\operatorname{Re}\left(J_{s}^{*}\right)$, and the dashed line $\operatorname{Im}\left(J_{s}^{*}\right)$ (circular nonmetallic inclusion).

depicted in Fig. 1(a,b) and $d_{p}$ is the depth of inclusion's or crack's tip from the surface. The self-inductance of the coil of per unit length in air without bar is $L_{o}=\mu_{o} n^{2} \pi R_{o}^{2}$, where $R_{o}$ is the radius of the coil's cross-section. According to the above expressions, $f^{*}$ can also be written as $f^{*}=2(R / \delta)^{2}$, and the ratio, $d / \delta$, is expressed as $d / \delta=d^{*} \sqrt{f^{*} / 2}$. The nondimensional impedance change in terms of the fill factor $\eta=\left(R / R_{o}\right)^{2}$ and the relative permeability $\mu_{r}$ of the metal bar is expressed as

$\Delta Z^{*}=-\eta \mu_{r} \int_{\partial \Omega_{1}} \Delta J_{s}^{*} \mathrm{~d} l^{*} / \pi f^{*}$.

By this treatment, the number of parameters for each problem is reduced to minimum. In calculating Eq. (20), the current density $J_{s}^{*}$ is determined by BEM, and $J_{s 0}^{*}$ of a flawless circular bar is given by an analytical solution [1]

$J_{s 0}^{*}=\alpha J_{1}(\alpha r / R) / J_{0}(\alpha)$,

where $\alpha=(1-j) \sqrt{f^{*} / 2}$.

In Eq. (21), $J_{0}, J_{1}$ are the Bessel function of the first kind of order zero and order one. According to Eq. (15), the impedance of the coil, with the flawless bar, is written as

$Z_{0}^{*}=j(1-\eta)-\eta \mu_{r} \frac{2 \alpha J_{1}(\alpha)}{f^{*} J_{0}(\alpha)}$.

\section{Numerical results and discussion}

The numerical method, BEM, is used to discretize the geometric boundary and physical unknowns for solving these integral equations. In the discretization, the boundary is divided into several segments, and each segment is an element which is a three-node, isoparameter, quadratic one for interpolation. The criterion for discretizing the boundary is that these nodes are distributed on the boundary as evenly as possible. Since the procedure of BEM is a standard one [22], no attempt is made to mention it in detail. For the inclusion, it is easy to deal with the numerical calculation. For the embedded crack, since there exist two crack tips, the behavior of eddy currents at these places can be described using special elements possessing a weak singularity [23], $1 / \sqrt{r}$, for the interpolation and extrapolation of BEM. When the collocation point $\mathbf{x}$ coincides with each node of discretization, one corresponding integral equation in the above is utilized. After the discretization and the integration are done, a set of algebraic equations are obtained in terms of the nodal values $J_{s}$ of $\partial \Omega_{1}$ and $\left[J_{s}\right]$, $H_{z}$ of $\partial \Omega_{c}$ as unknowns for cracked problems, and can be easily solved by a numerical method, e.g. LU decomposition. When obtaining the surface current densities, we substitute them into the integral representation of impedance (Eq. 16), and use the above procedure of discretization and integration to determine the impedance value of the coil.

\subsection{Circular bar with a nonmetallic inclusion}

In the following, we only present the numerical results for the circular inclusion with $\mu_{r 2}=1$. To solve this problem, some collocation points are chosen on the boundary $\partial \Omega=\partial \Omega_{1} \cup \partial \Omega_{2}$, and these points also correspond to the nodes of discretization. No matter if the collocation points are on $\partial \Omega_{1}$ or on $\partial \Omega_{2}$, either Eq. (9) or Eq. (10) can be used to combine with Eq. (11) as the governing equations in terms of $J_{s}^{*}(\mathbf{x}), \mathbf{x} \in \partial \Omega_{1} \cup \partial \Omega_{2}$. Fig. 2(a) shows the distribution of current density on the circumference of a bar with a circular inclusion for $f^{*}=5, d^{*}=0.2, d_{p}^{*}=0.1$, and Fig. 2(b) for the distribution on the boundary of inclusion. In Fig. 2 , the corresponding result of normalized non-dimensional impedance change is $\Delta Z^{*} / \eta \mu_{r},=\left(-2.403 \times 10^{-3}\right.$, $\left.1.003 \times 10^{-2}\right)$, and the constant magnetic field in the 


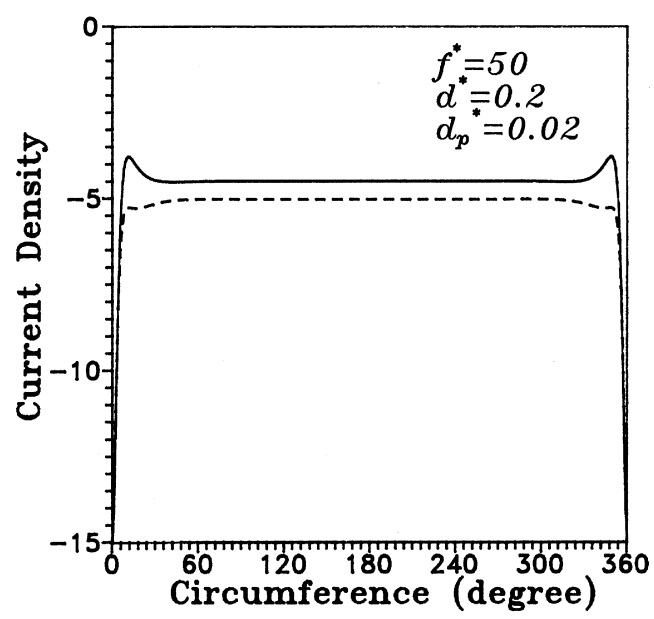

(a)

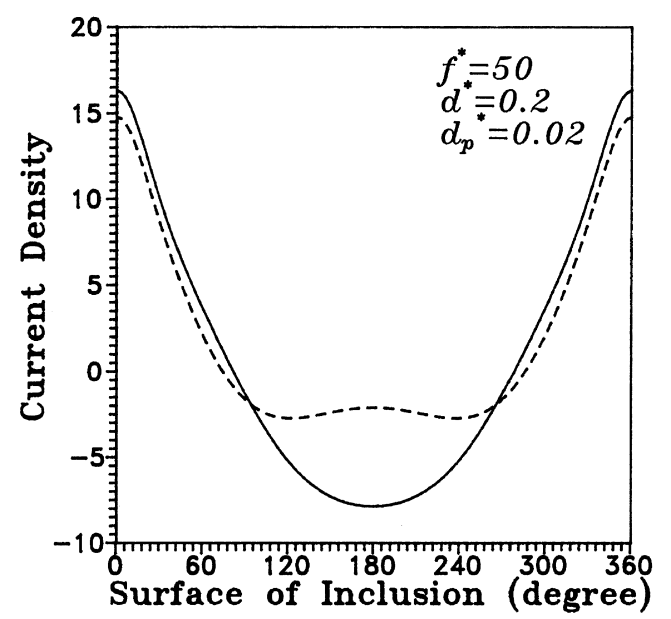

(b)

Fig. 3. (a) Distribution of $J_{s}^{*}$ on circumference $\partial \Omega_{1}$ for $f^{*}=50, d^{*}=0.2$, $d_{p}^{*}=0.02 . H_{z}^{(2) *}=(0.692,-0.285), \Delta Z^{*} / \eta \mu_{r}=\left(5.710 \times 10^{-3}, 8.236 \times\right.$ $\left.10^{-3}\right)$. The solid line denotes $\operatorname{Re}\left(J_{s}^{*}\right)$, and the dashed line $\operatorname{Im}\left(J_{s}^{*}\right)$ (circular nonmetallic inclusion). (b) Distribution of $J_{s}^{*}$ on the surface of the inclusion. The solid line denotes $\operatorname{Re}\left(J_{s}^{*}\right)$, and the dashed line $\operatorname{Im}\left(J_{s}^{*}\right)$ (circular nonmetallic inclusion). inclusion is $H_{z}^{(2) *}=(0.838,-0.260)$. The numerical results for a case with $f^{*}=50, d^{*}=0.2, d_{p}^{*}=0.02$ are plotted in Fig. 3(a,b). The current densities almost keep constant along the circumference, but change abruptly in the vicinity of a sub-surface defect, as indicated in Fig. 2(a) and Fig. 3(a). Moreover, the closer the inclusion is to the circumference, the higher the current density is in the gap between them. Generally, a lower frequency is adopted for detecting deeper embedded defects, due to the skin effect of the eddy current. If the circular inclusion is at the center of a circular bar, there is an available analytical solution for this axisymmetric problem. Table 1 lists the results, $J_{s}^{*}, H_{z}^{(2) *}$, of analytical and BEM solutions at $f^{*}=5,50$ for comparison. From Table 1, one can observe that the results of BEM reasonably agree with analytical solutions. Hence the accuracy of BEM is verified for the bars with a nonmetallic inclusion. Figs. 4 and 5 show the impedance changes of a coil by the circular inclusion for various lengths and depths at $f^{*}=5,50$, respectively.

\subsection{Circular bar with an embedded crack}

Either Eq. (12a) or Eq. (13a) is used to combine with Eq. (12b) and Eq. (14) or BEM formulation with unknowns$J_{s}^{*}(\mathbf{x}), \mathbf{x} \in \partial \Omega_{1},\left[J_{s}^{*}(\mathbf{x})\right], H_{z}^{(c) *} \mathbf{x} \in \partial \Omega_{c}^{+}$. Once the surface currents are gained, substituting them into Eq. (13b) one can further determine $J_{s}^{-}, J_{s}^{+}$on the crack surface. Brudar [19] calculated a case, $f^{*}=5, d^{*}=1 / 3, d_{p}^{*}=1 / 6, \theta=90^{\circ}$, but only presented the distribution of the magnetic field in the circular bar. For this case, our results for the constant magnetic field at the crack, $H_{z}^{(c) *}=(0.7,-0.4)$, quite agree with his. In Fig. 6, we depict the current distribution on the circumference and crack surface for $f^{*}=5, d^{*}=0.2$, $d_{p}^{*}=0.1, \theta=90^{\circ}$. The results of $f^{*}=50, d^{*}=0.2$, $d_{p}^{*}=0.02, \theta=90^{\circ}$ are plotted in Fig. 7. The cases with a perpendicular crack, $\theta=90^{\circ}$, are symmetrical problems, so the distributions of surface current on both sides of the crack are the same, i.e. $J_{s}^{-}=J_{s}^{+}$. Moreover, when the crack is located at the center of the circular bar, symmetry of current exists not only on the upper and lower side of the crack but also on the right-hand and left-hand side, due to the geometric symmetry. Fig. 8 illustrates this symmetry for $f^{*}=5, d^{*}=0.6, d_{p}^{*}=0.7, \theta=90^{\circ}$. Since there is nearly

Table 1

Comparison of analytical and BEM solutions for a circular inclusion at the center of a bar. $J_{s}^{O *}$ : the current density along the circumference of a bar. $J_{s}^{I^{*}}$ : the current density along the circular inclusion

\begin{tabular}{|c|c|c|c|c|c|}
\hline & & \multicolumn{2}{|l|}{$d^{*}=1.0, d_{p}^{*}=0.5$} & \multicolumn{2}{|l|}{$d^{*}=0.5, d_{p}^{*}=0.75$} \\
\hline & & Analytical & BEM & Analytical & BEM \\
\hline \multirow[t]{3}{*}{$f^{*}=5$} & $H_{z}^{(2) *}$ & $(0.536,-0.580)$ & $(0.536,-0.580)$ & $(0.391,-0.639)$ & $(0.391,-0.639)$ \\
\hline & $J_{s}^{O *}$ & $(-0.936,-1.807)$ & $(-0.936,-1.807)$ & $(-0.923,-1.754)$ & $(-0.923,-1.754)$ \\
\hline & $J_{s}^{I *}$ & $(0.725,0.670)$ & $(0.725,0.669)$ & $(0.400,0.244)$ & $(0.400,0.243)$ \\
\hline \multirow[t]{3}{*}{$f^{*}=50$} & $H_{z}^{(2) *}$ & $(-8.816 \mathrm{E}-2,-1.597 \mathrm{E}-2)$ & $(-8.816 \mathrm{E}-2,-1.597 \mathrm{E}-2)$ & $(-3.331 \mathrm{E}-2,3.987 \mathrm{E}-2)$ & $(-3.331 \mathrm{E}-2,3.987 \mathrm{E}-2)$ \\
\hline & $J_{s}^{O *}$ & $(-4.446,-5.027)$ & $(-4.446,-5.027)$ & $(-4.489,-5.017)$ & $(-4.489,-5.017)$ \\
\hline & $J_{s}^{I *}$ & $(0.200,-1.102)$ & $(0.199,-1.102)$ & $(-0.249,-0.208)$ & $(-0.249,-0.208)$ \\
\hline
\end{tabular}




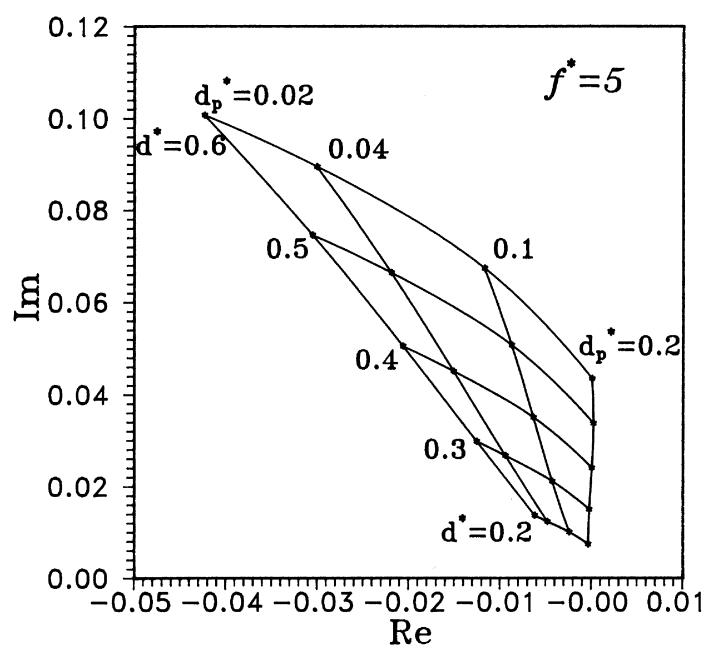

Fig. 4. Impedance change of the coil $\Delta Z^{*} / \eta \mu_{r}$ vs. various $d^{*}$ and $d_{p}^{*}$ for $f^{*}=5$ (circular nonmetallic inclusion).

no perturbation of current density on the circumference, as shown in Fig. 8(a), it implies ECT is not sensitive to a deeper crack, according to Eq. (16). Fig. 9 shows the asymmetric case, $\theta=60^{\circ}$, for $f^{*}=5, d^{*}=0.2$, $d_{p}^{*}=0.1$. In the above cases, the numerical results are consistent with the assumption, $H_{z}^{(c) *}$ is a finite value in a closed crack, leading to Eq. (14). From these figures, we also observe that the surface current along the circumference almost remains constant until near the crack tip, then changes abruptly. In addition, the current density approaches to infinite at the crack tips, in which the asymptotic behavior is $1 / \sqrt{r}$ [23], as indicated in these figures. Here, we have to mention that the cross-section of metal with contour, $\partial \Omega=\partial \Omega_{1} \cup$ $\partial \Omega_{c}$, is simply-connected for a surface-breaking crack problem [16,17], while is multiply-connected for an embedded crack problem. This leads to some significant differences of current distribution in the two problems.

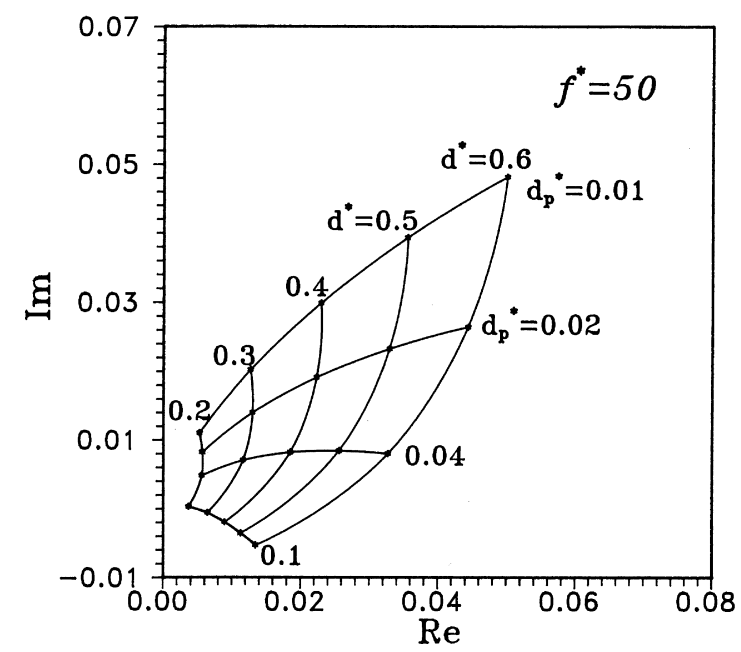

Fig. 5. Impedance change of the coil $\Delta Z^{*} / \eta \mu_{r}$ vs. various $d^{*}$ and $d_{p}^{*}$ for $f^{*}=50$ (circular nonmetallic inclusion).

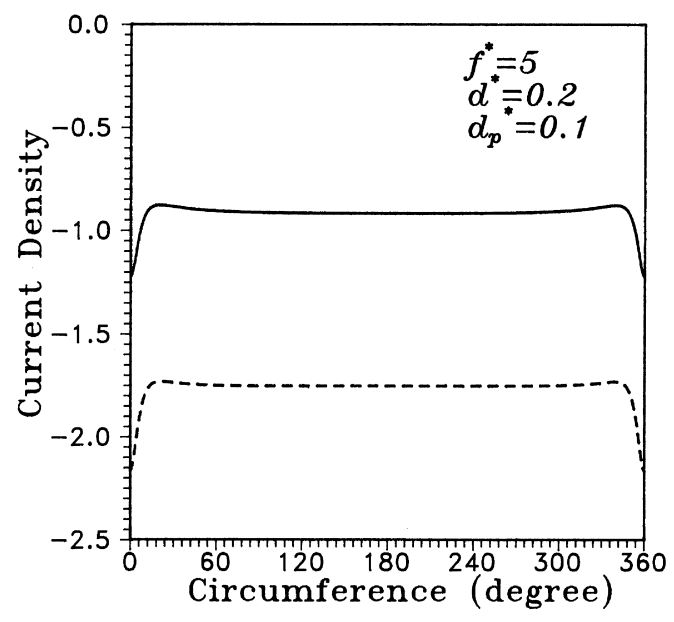

(a)

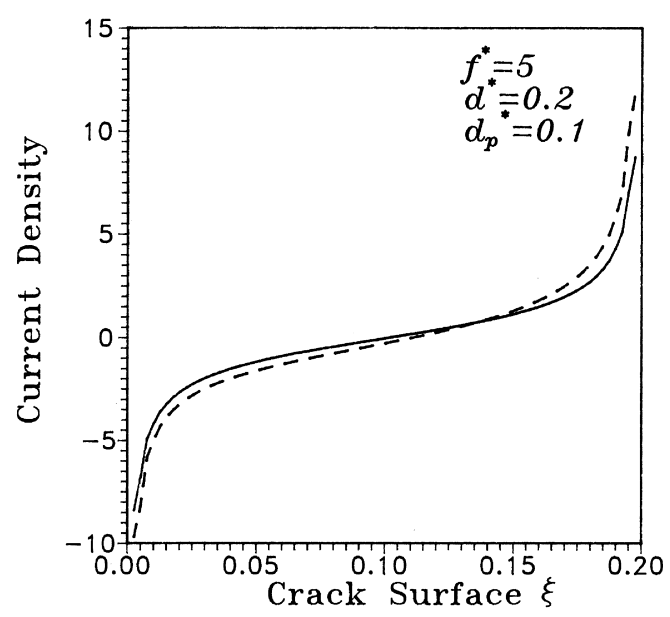

(b)

Fig. 6. (a) Distribution of $J_{s}^{*}$ on circumference $\partial \Omega_{1}$ for $f^{*}=5, d^{*}=0.2$, $d_{p}^{*}=0.1, \theta=90^{\circ} . H_{z}^{(2) *}=(0.821,-0.274), \Delta Z^{*} / \eta \mu_{r}=\left(-9.984 \times 10^{-4}\right.$, $\left.4.759 \times 10^{-3}\right)$. The solid line denotes $\operatorname{Re}\left(J_{s}^{*}\right)$, and the dashed $\operatorname{line} \operatorname{Im}\left(J_{s}^{*}\right)$ (embedded crack); (b) Distribution of $\left[J_{s}^{*}\right]$ on the surface of the crack. The solid line denotes $\operatorname{Re}\left(\left[J_{s}^{*}\right]\right)$, and the dashed line $\operatorname{Im}\left(\left[J_{s}^{*}\right]\right)$ (embedded crack).

For the embedded crack, there are two crack tips, so current can flow around the crack. Since the crack is an obstacle for eddy current, there is a stagnation zone, where the amplitude of current density nearly vanishes, in the middle of the embedded crack, as shown in Fig. 6(b) and Fig. 7(b). When the depth of a sub-surface crack decreases, the current density within the narrow gap, between the crack tip and the circumference, increases. Whereas, if the depth of the crack is zero, i.e. the surface-crack problem, the crack connects with the circumference, and there is only one crack tip for this simple-connected problem. Therefore, on the two sides of the mouth of crack, there are two corners which are dead zones for current, so current cannot flow through the mouth, and only along the crack from one side to the other, passing by the crack tip 


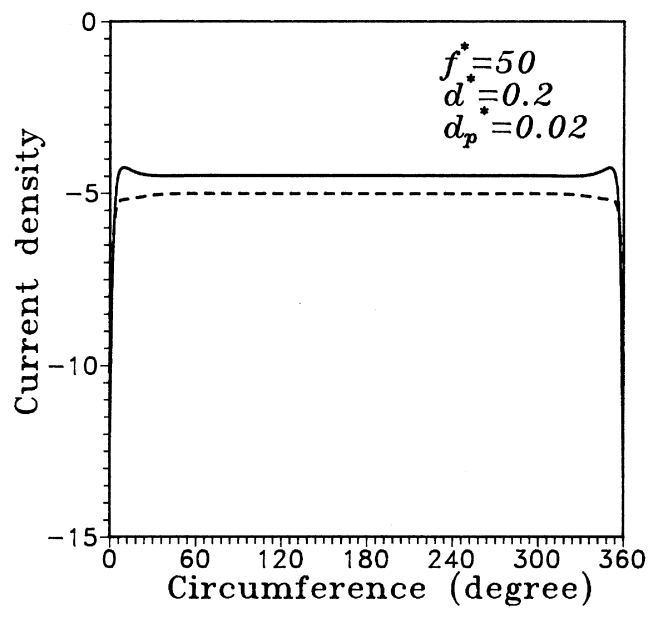

(a)

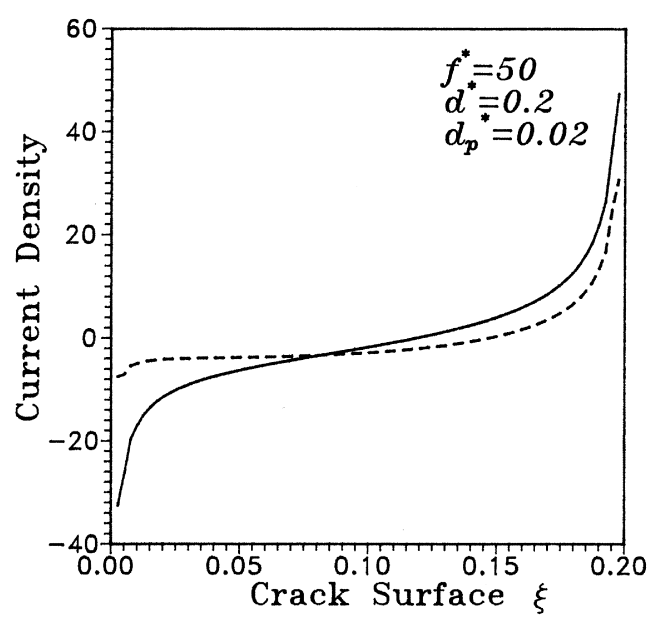

(b)

Fig. 7. (a) Distribution of $J_{s}^{*}$ on circumference $\partial \Omega_{1}$ for $f^{*}=50, d^{*}=0.2$, $d_{p}^{*}=0.02, \theta=90^{\circ} . H_{z}^{(c) *}=(0.593,-0.289), \Delta Z^{*} / \eta \mu_{r}=\left(2.782 \times 10^{-3}\right.$, $\left.2.780 \times 10^{-3}\right)$. The solid line denotes $\operatorname{Re}\left(J_{s}^{*}\right)$, and the dashed line $\operatorname{Im}\left(J_{s}^{*}\right)$ (embedded crack); (b) Distribution of $\left[J_{s}^{*}\right]$ on the surface of the crack. The solid line denotes $\operatorname{Re}\left(\left[J_{s}^{*}\right]\right)$, and the dashed line $\operatorname{Im}\left(\left[J_{s}^{*}\right]\right)$ (embedded crack).

[23]. Another difference is that for the embedded crack the line integral of current along the crack equals zero, Eq. (14), while for surface-breaking crack, it is not equal to zero. Under the same conditions, $d^{*}, d_{p}^{*}, f^{*}$, it is also observed that the influence of a nonmetallic inclusion on the eddy current perturbation is more significant than that of a crack by comparing Fig. 2(a) with Fig. 6(a), and Fig. 3(a) with Fig. 7(a).

After surface currents are obtained by BEM, the normalized non-dimensional impedance changes, $\Delta Z^{*} / \eta \mu_{r}$, can be calculated subsequently by using Eq. (20). The results for the perpendicular crack, $\theta=90^{\circ}$, against the crack length and depth are shown in Figs. 10 and 11 for $f^{*}=5,15$, respectively. The tendency that impedance change is located in the second quadrant, when $f^{*}$ is lower, is observed from these figures.

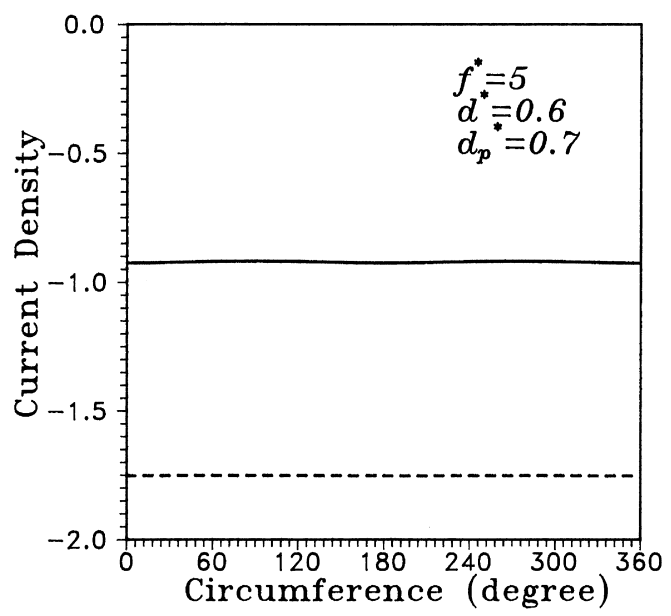

(a)

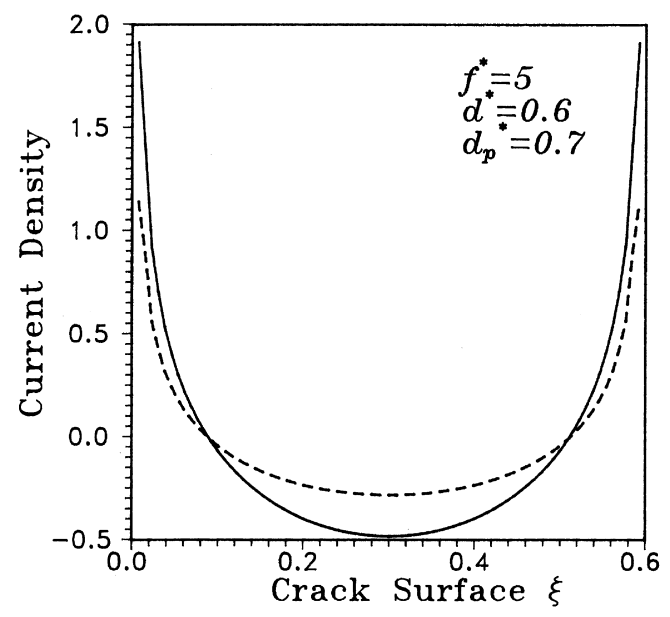

(b)

Fig. 8. (a) Distribution of $J_{s}^{*}$ on circumference $\partial \Omega_{1}$ for $f^{*}=5, d^{*}=0.6$, $d_{p}^{*}=0.7, \theta=90^{\circ} . H_{z}^{(c) *}=(0.376,-0.645), \Delta Z^{*} / \eta \mu_{r}=\left(3.538 \times 10^{-4}\right.$, $\left.5.957 \times 10^{-4}\right)$. The solid line denotes $\operatorname{Re}\left(J_{s}^{*}\right)$, and the dashed line $\operatorname{Im}\left(J_{s}^{*}\right)$ (embedded crack); (b) Distribution of $\left[J_{s}^{*}\right]$ on the surface of the crack. The solid line denotes $\operatorname{Re}\left(\left[J_{s}^{*}\right]\right)$, and the dashed line $\operatorname{Im}\left(\left[J_{s}^{*}\right]\right)$ (embedded crack).

\section{Conclusion}

In this paper, we had shown the application of BEM for solving surface integral equations is useful for bars with a sub-surface crack or nonmetallic inclusion. Since the magnetic field, a constant, is unknown in the embedded flaw, we employ a necessary integral equation as the inner boundary condition to combine with the governing integral equations of the bar for solving the current and magnetic field. With the aid of an extra auxiliary surface integral equation, the eddy current distribution around the crack can be described clearly. In addition, we also calculate the impedance changes of the coil in terms of surface current vs. the length, depth and orientation of the embedded flaw. The results of the numerical simulation enable us to select an optimal frequency to inspect bars by encircling-type probes, 


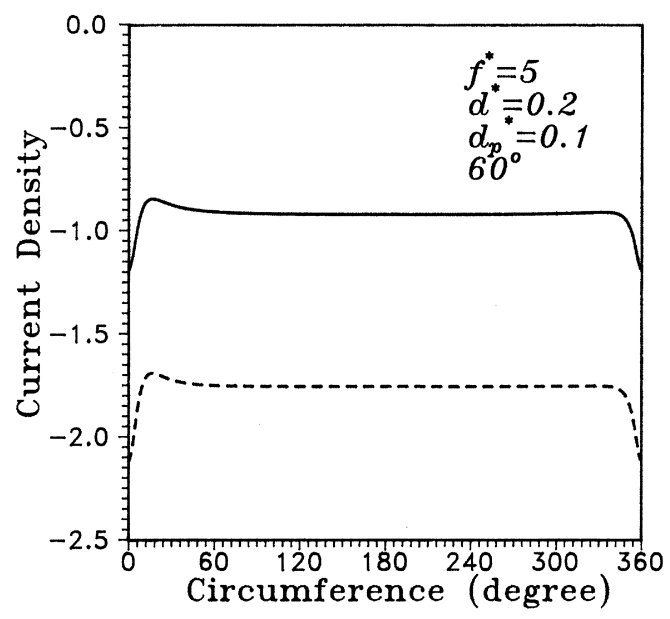

(a)

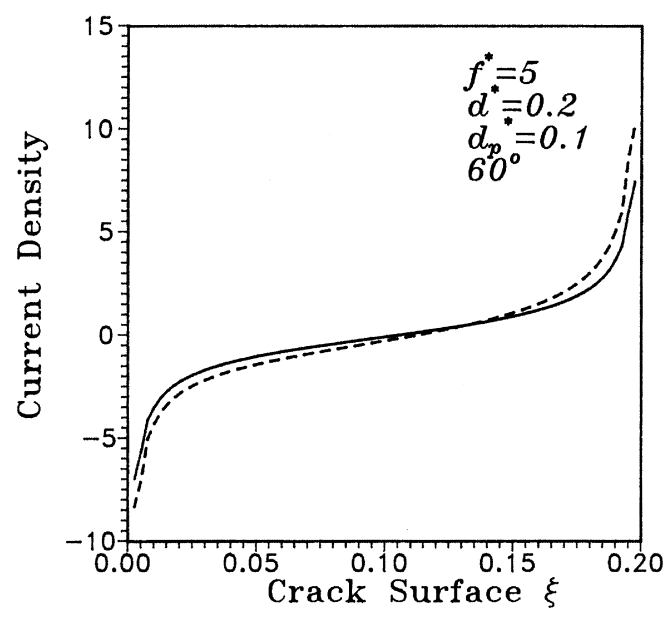

(b)

Fig. 9. (a) Distribution of $J_{s}^{*}$ on circumference $\partial \Omega_{1}$ for $f^{*}=5, d^{*}=0.2$, $d_{p}^{*}=0.1, \theta=60^{\circ} . H_{z}^{(c) *}=(0.832,-0.259), \Delta Z^{*} / \eta \mu_{r}=\left(-8.158 \times 10^{-4}\right.$, $\left.3.440 \times 10^{-3}\right)$. The solid line denotes $\operatorname{Re}\left(J_{s}^{*}\right)$, and the dashed line $\operatorname{Im}\left(J_{s}^{*}\right)$ (embedded crack); (b) Distribution of $\left[J_{s}^{*}\right]$ on the surface of the crack. The solid line denotes $\operatorname{Re}\left(\left[J_{s}^{*}\right]\right)$, and the dashed line $\operatorname{Im}\left(\left[J_{s}^{*}\right]\right)$ (embedded crack).

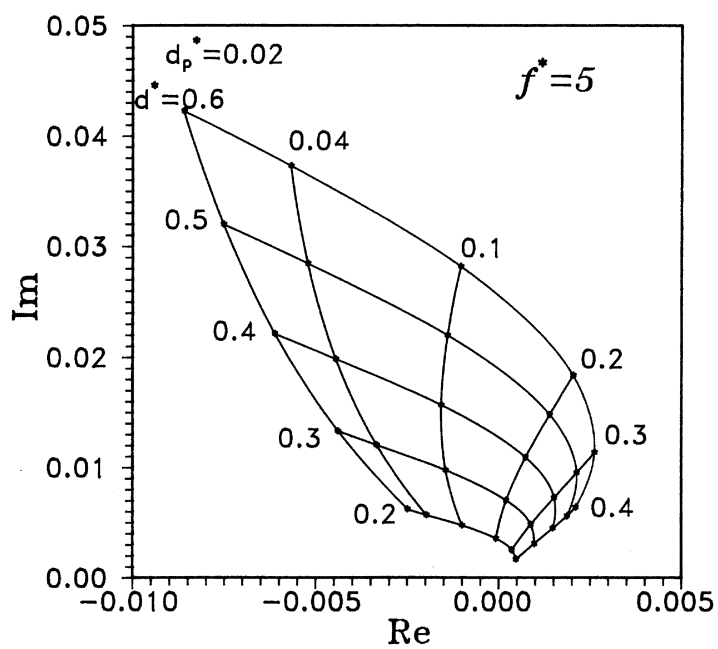

Fig. 10. Impedance change of coil $\Delta Z^{*} / \eta \mu_{r}$ vs. various $d^{*}$ and $d_{p}^{*}$ for $f^{*}=5$ (embedded crack).

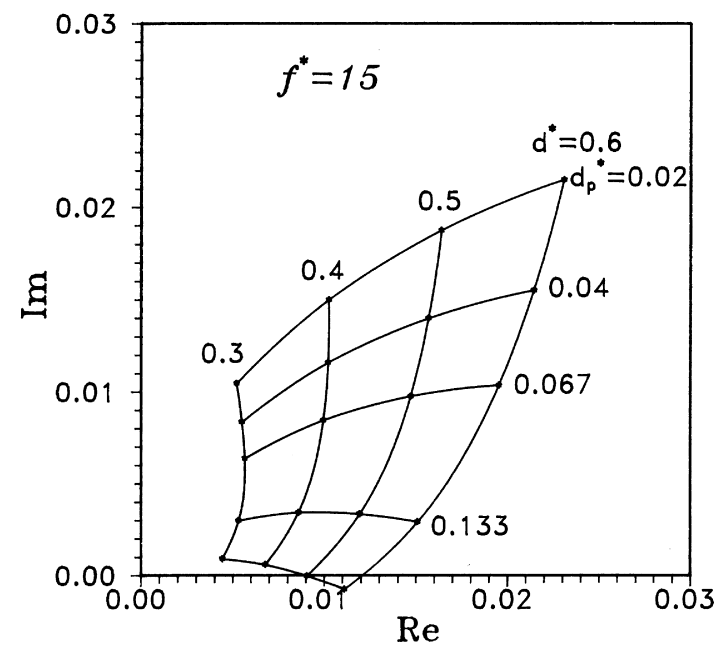

Fig. 11. Impedance change of coil $\Delta Z^{*} / \eta \mu_{r}$ vs. various $d^{*}$ and $d_{p}^{*}$ for $f^{*}=$ 15 (embedded crack).

and also offer an interpretation about the characteristics of flaws for ECT measurements. We do not intend to extend these problems to complicated cases in this paper. However, all the above formulations can be applied for 2-D multi-flaw problems.

The foregoing method also has a potential for the application of infra-red scanning billet, in which the coil induces eddy currents to generate heat [3], and the infra-red sensors scan the temperature difference to detect the defects. When the surface current is determined by BEM, one can use the integral representation of current to calculate the current density field, which is a heat source, in the billet. Then combining FEM to calculate the temperature field on the surface of the billet, one can estimate the results of thermography for the inspection of the billet.

\section{Acknowledgements}

This research is sponsored by the Ministry of Economic Affairs, Taiwan, ROC. All the calculations were performed by the alpha computer of the Materials Research Labs., Industrial Technology Research Institute.

\section{References}

[1] Forster F. Electromagnetic testing. Nondestructive testing handbook, 2 ed., vol. 4. 1986:113-45.

[2] Mukherjee S, Morjaria MA, Moon FC. Eddy current flows around cracks in thin plates for nondestructive testing. ASME J Appl Mech 1982;49:389-95.

[3] Muhlbauer A, Muiznieks A, Leßmann HJ. The calculation of 3D high-frequency electromagnetic fields during induction heating using the BEM. IEEE Trans Mag 1993;29(2):1566-9.

[4] Lord W, Nath S, Shin YK, You Z. Electromagnetic methods of defect detection. IEEE Trans Mag 1990;26(5):2070-5.

[5] Bowler JR. Eddy current interaction with an ideal crack. I. The forward problem. J Appl Phys 1994;75(12):8128-37. 
[6] Beissner RE. Scalar potential model of eddy-current interactions with three-dimensional flaws. J Nondestructive Eval 1988;7:15-24.

[7] Beissner RE. Approximate model of eddy-current probe impedance for surface-breaking flaws. $J$ Nondestructive Eval 1988;7:25-34.

[8] Burke SK. Eddy-current inversion in the thin-skin limit: determination of depth and opening for a long crack. J Appl Phys 1994;76(5):3072-80.

[9] Michael DH, Lewis AM, McIver M, Collins R. Thin-skin electromagnetic fields in the neighborhood of surface-breaking cracks in metals. Proc R Soc Lond A 1991;434:587-603.

[10] Hashizume H, Yamada Y, Miya K, Toda S, Morimoto K, Araki Y, Satake K, Shimizu N. Numerical and experimental analysis of eddy current testing for a tube with cracks. IEEE Trans Mag 1992;28(2):1469-72.

[11] Hower GL, Oslen RG, Philipp LD, Staley JE. A method for predicting the response to three-dimensional defects in eddy current NDE. Mater Eval 1982;40:1362-6.

[12] Beissner RE. Boundary element model of eddy current flaw detection in three dimensions. J Appl Phys 1986;60(1):352-6.

[13] Kahn AH. Boundary integral equation methods for two-dimensional models of crack-field interactions. J Nondestructive Eval 1988;7:314.

[14] Bowler JR. Eddy current field theory for a flawed conducting half-space. In: Thompson DO, Chimenti DE, editors. Review of progress in quantitative nondestructive evaluation, vol. V, 1986:149-55.

[15] Dodd CV, Deeds WE. Analytical solutions to eddy-current probe-coil problems. J Appl Phys 1968;39:2829-38.

[16] Spal R, Kahn AH. Eddy currents in a conducting cylinder with a crack. J Appl Phys 1979;50:6135-8.

[17] Kahn AH, Spal R. A boundary integral equation method for calculating the eddy-current distribution in a long cylindrical bar with a crack. In: Birnhaum G, Free G, editors. Eddy-current characterization of materials and structures. ASTM STP 722, 1981:299-307.

[18] Chari MVK, Kincaid TG. Finite-element analysis of eddy-current flaw detection. In: Birnhaum G, Free G, editors. Eddy-current characterization of materials and structures. ASTM STP 722, 1981:57-75.

[19] Brudar B. The electromagnetic field in the neighborhood of a defect in a material. In: Sharpe RS, editor. Research techniques in nondestructive testing, vol. V, 1982:170-238.

[20] Stratton A. Electromagnetic theory. New York: McGraw-Hill, 1941:464-7.

[21] Auld BA, Muennemann F, Winslow DK. Eddy current probe response to open and closed surface flaws. J Nondestructive Eval 1981;2:1-21.

[22] Brebbia CA et al. Boundary element techniques. Berlin: SpringerVerlag, 1984.

[23] Kahn AH, Spal R, Feldman A. Eddy-current losses due to a surface crack in conducting material. J Appl Phys 1977;48:4454-9. 